\title{
NETWORKS OF INTERACTING STOCHASTIC FLUID MODELS
}

\author{
NIKKI SONENBERG
}

(Received 26 July 2018; first published online 30 August 2018)

2010 Mathematics subject classification: primary 60J20; secondary 90B15.

Keywords and phrases: stochastic fluid models, ad hoc networks, matrix analytic methods, finite buffers.

Stochastic fluid models have been widely used to model the level of a resource that changes over time, where the rate of variation depends on the state of some continuous time Markov process. Stochastic fluid models are two-dimensional Markov processes in which the first component, the fluid level, is continuous and the second component, the phase, is discrete. The focus of this thesis is the study of networks of interacting stochastic fluid models, with the motivating application being an ad hoc telecommunications network. Nodes represent communication devices and the fluid level at a node represents the amount of a resource, for example energy, available to that device.

Nodes communicate via multi-hop routes and rely on energy sources, for example batteries, to transmit within a given radius, from which the routes are defined. The energy consumption of a node is a function of its communication activity, and consumption rates may vary according to whether a node is a source, transit, or destination of a call. Latouche and Taylor [1] introduced an approach for analysing such networks of fluid models, each modulated by the same background process, which incorporates feedback from the fluid level at a node to the background phase process.

Assuming that the battery at the node can be recharged, they studied the energy resource required to ensure that the network can operate with a reasonable quality of service. They established criteria under which the network behaves as a loss network, that is, a network of links each of which is capacity constrained and where calls unable to be accepted are lost.

A contribution of this thesis is to extend the Latouche and Taylor analysis of a network with infinite buffers at each node to the situation where the buffers are finite.

Thesis submitted to The University of Melbourne in October 2017; degree approved on 21 March 2018; supervisor Peter Taylor.

(C) 2018 Australian Mathematical Publishing Association Inc. 
We derive the form of the stationary density using a Markov regenerative approach for the finite and infinite buffer cases, for which the latter contains new results. Of significance to the analysis of the finite model at a network node is the understanding that the stationary density can be expressed in a way that effectively separates the behaviour of the process at the boundaries from its behaviour in the interior. So the description of the stationary density of the finite buffer model rests heavily on the expression for the stationary density of the infinite buffer model, which we derive in a way that sets out all the steps of the fluid model analysis, and which integrates ideas from several papers in a consistent form.

For the network model, a reduced load approach is used to develop approximations accounting for interactions between nodes that occur because an empty buffer at a node can lead to the dropping of calls in the network. We illustrate the approach by deriving performance measures, such as the dropout probabilities for a simple network as characteristics such as buffer size are varied, and provide some insight into ways in which a battery charging process might be set so that it produces specific quality of service outcomes. Our results also provide insight into situations where the infinite buffer model is a reasonable approximation to the finite buffer model.

Since ad hoc networks rely on transit nodes, and because data forwarding consumes valuable battery energy, each node along the path has an inherent disincentive to cooperate. We investigate a mechanism introduced by Latouche and Taylor to promote collaboration throughout the network that involves a network with two fluid models at each node, one with energy and another with credit. A contribution of this thesis is to consider call blocking behaviour in such networks, which we obtain by drawing on the results derived for the stationary density of finite buffer models with varied behaviour at the boundaries.

\section{Reference}

[1] G. Latouche and P. G. Taylor, 'A stochastic fluid model for an ad hoc mobile network', Queueing Syst. 63 (2009), 109-129.

NIKKI SONENBERG, School of Mathematics and Statistics,

The University of Melbourne, Victoria 3010, Australia

e-mail: nikki.sonenberg@unimelb.edu.au 